\title{
Sustainability Topics in Physics Education, Science Agency Beliefs and Physics Identity
}

\author{
John Christopher Doscher*, Zahra Hazari ${ }^{\dagger}$, Geoff Potvin ${ }^{\ddagger}$ and Leidy Klotz ${ }^{\S}$ \\ *Florida International University
${ }^{\dagger}$ Department of Teaching and Learning, Department of Physics and STEM Transformation Institute, \\ Florida International University \\ ¿Department of Physics and STEM Transformation Institute, Florida International University, \\ 11200 SW $8^{\text {th }}$ St., Miami, FL 33199 \\ ${ }^{\S}$ Glenn Department of Civil Engineering, Clemson University, Lowry Hall, Clemson, SC 29634
}

\begin{abstract}
The physics classroom provides an excellent opportunity for students to comprehend global sustainability issues and simultaneously be empowered by their science learning. Drawing on data from a large national survey study of college students about their high school science experiences, we compare how frequently sustainability topics (e.g. energy supply, energy demand, climate change, water supply) are covered in high school physics versus other science courses. Furthermore, employing science agency and physics identity theoretical perspectives, we examine whether the inclusion of sustainability topics has an effect on student science agency beliefs or their physics identities while controlling for demographics and other background variables. Our results indicate that physics courses are reported to cover sustainability topics less frequently than other science courses. In addition, the inclusion of certain topics (e.g. energy supply) has significant effect on student science agency beliefs and physics identity.
\end{abstract}

PACS: 01.40.ek, 01.40.Fk, 01.75.+m

\section{INTRODUCTION}

"The greatest shortcoming of the human race is our inability to understand the exponential function." [1]

As the global environment changes, there is a growing need for students to have a better understanding of issues related to global sustainability. Physics and STEM education in general, fulfill crucial roles in helping students to make responsible decisions with regards to sustainable actions on personal, community, and policy levels as citizens of the Earth and further to contribute to future advances and solutions that will help preserve the long term welfare of human society (e.g. through STEM careers). Students need to develop a better scientific understanding of global sustainability issues, which include physics and mathematics topics (e.g. energy, thermodynamics, exponential function). In the context of physics courses, this serves another purpose: empowering students to learn physics since they have been found to perceive physics to be narrow and disconnected from their lives [2].

Much of recent science education research has focused on empowering students in their science learning by increasing their level of input as well as crossing boundaries from the world of the classroom to a more relevant social and physical world outside the classroom [3]. We believe that this is an important direction for physics education though it may be more difficult in physics than the other sciences since the physics curriculum is driven by a traditional canon of knowledge. It is even more pertinent that we begin to shift our emphasis in physics education from maintaining the canon, to helping students understand the physical world around them in ways that are meaningful to them and their future, as well as meaningful for the future of scientific progress. Sustainability topics may also be important in helping to diversify physics since topics with social relevance have been found to be more attractive to particular underrepresented groups in physics such as women [4]. This work focuses on understanding how sustainability topics can be used to cultivate science agency beliefs and physics identity. That is to understand how knowledge about sustainability topics can promote a sense of ability to positively affect the world, garner recognition, pursue personal interests, and feel competent about taking action.

\section{BACKGROUND}

The colloquial term sustainability applies to an activity or function intended to continue "for an unspecified long period of time" [5]. As a more formal topic of study, it refers to creating and maintaining the conditions under which humans and nature can exist in productive harmony, that permit fulfilling the social, economic and other requirements of present and future generations [6]. Most topics related to sustainability are addressed in environmental science courses rather than biology, chemistry, and physics [7]. A drawback in teaching sustainability topics through environmental science courses is the limited numbers of students who take such courses. Environmental science courses in high school are normally elective courses taken by a select group already predisposed to thinking about such issues. Sustainability topics and the associated issues are central to our future and the future of life on Earth. It is 
important that more students in general have the opportunity to engage in thinking deeply and become motivated to act in environmentally, socially, and economically responsible ways. The application of science is a crosscutting concern and it is likely that many sustainability topics are already being discussed within high school science courses, however little information exists as to which topics are being covered and how addressing these topics affect student attitudes. There is a need to address sustainability topics across the science curriculum and reassess how high school physics courses, one of the last opportunities to engage many students with physics content, can be more empowering and meaningful for students.

Basu and Barton refer to "critical subject agency" in preparing students to act on injustice in their lives. Here we focus on "critical science agency" as the belief, that is the perception of oneself, as being able to affect the world through science knowledge [8]. We define physics identity as the amalgamation of three factors: recognition, competence/performance, and interest [9]. Recognition results from being perceived by others as a good physics student. Competence/performance is the confidence that physics tasks can be completed and comprehended. Interest is the motivation to pursue understanding physics with some depth and study.

This work addresses the following research questions and as a result aims to provide an impetus for including sustainability topics in physics education that enhance student science agency beliefs and physics identity:

(1) How frequently are sustainability topics included in physics courses compared to other science courses?

(2) Does inclusion of sustainability in physics courses predict students' science agency beliefs?

(3) Does inclusion of sustainability in physics courses predict students' physics identity?

\section{DATA COLLECTION AND METHODS}

The Sustainability and Gender in Engineering (SaGE) (NSF Grant Number 1036617) survey (http://stem.fiu.edu/sage) was conducted in fall of 2011. The participating schools were recruited from a stratified random sample of a comprehensive list of all U.S. postsecondary institutions. In total, 6772 students from both STEM and non-STEM majors returned surveys, with representation from each of the 50 recruited institutions (100\% institutional response rate). The SaGE survey included items that probed high school experiences, particularly in math and science courses, attitudes and beliefs about the role of science and engineering in the modern world, self-ascribed science and math identities, and demographic information. In order to address research question (1) the frequency of student responses from the survey on sustainability topic coverage in high school physics, chemistry, and biology courses was used. Multiple linear-regression was applied to the same response data to address research questions (2) and (3). The regressions included controls for race, ethnicity, gender, and an Academic Achievement Index (AAI) variable that assessed the totality of prior academic performance, including physics grades. Family support of science, parental education level, and the extracurricular STEM activities of respondents were also controlled for in the model to account for alternative hypotheses. Beyond the control variables, the key predictor variables that addressed questions (2) and (3) in the regression were the sustainability topic items. The outcome variables were science agency (2) and physics identity (3). Throughout this analysis, the maximum allowed chance of Type I error, was fixed at 5\%. All data analysis was conducted using R and utilized the "car" and "psych" packages [10]. There is a self-report limitation to the survey. However, a test-retest reliability study with 62 students revealed that the survey responses were stable with a reliability coefficient over 0.7 .

\section{RESULTS}

The results of the SaGE survey for students reporting sustainability topic coverage in physics courses $(n=2625)$ indicated that energy demand was covered more than in chemistry $(n=3972)$ and biology $(n=4527)$ courses. Energy supply was covered less than in both chemistry and biology. All other sustainability topics were covered more in other courses than in physics. In Fig. 1 the percentage of respondents reporting that they were exposed to specific sustainability topics in high school physics, chemistry, and/or biology is displayed.

\section{A. Controls}

Multiple regression analysis with science agency beliefs as the outcome yielded significant positive relationships for extracurricular STEM activity, and family interest in science.

Multiple regression analysis with physics identity as the outcome yielded significant positive relationships for science AAI, extracurricular STEM activity, and family interest. A significant negative relationship was found for gender and English AAI with physics identity. 
Figure 1. Frequency of students reporting sustainability related topic coverage in high school science courses

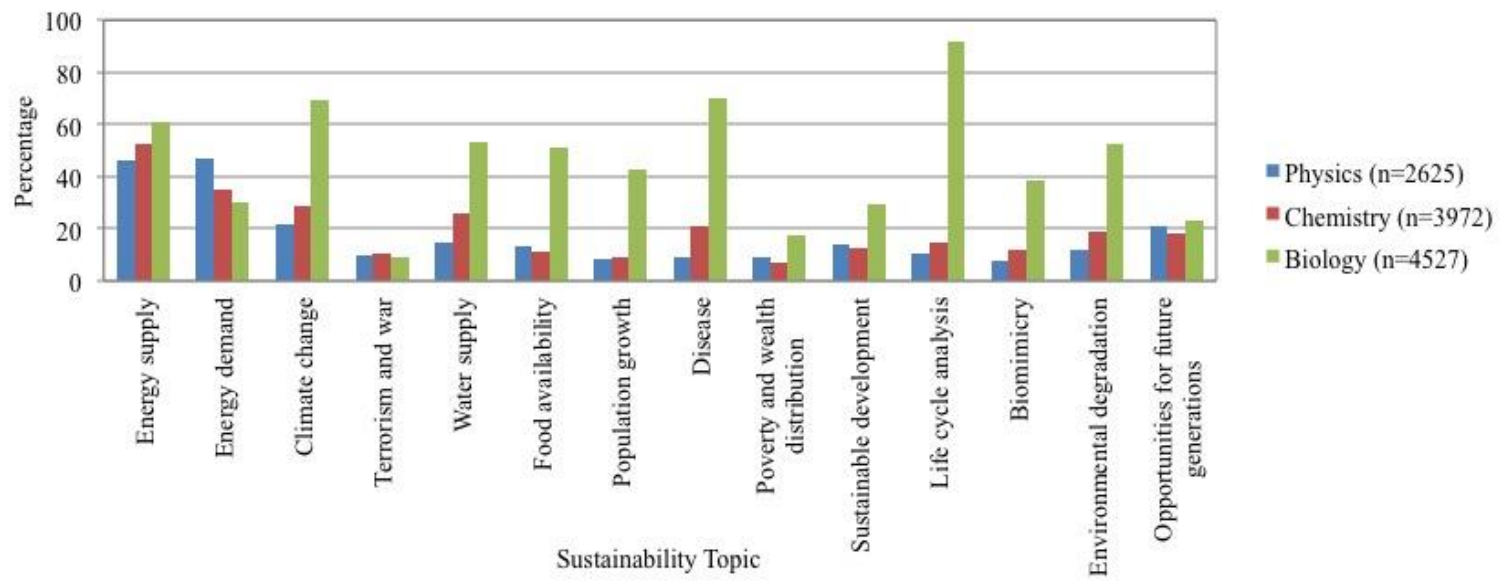

Table I. Multiple regression model predicting agency beliefs with sustainability-related topics in high school physics

\begin{tabular}{llll}
\hline Parameters & Estim. & Sig. & SE \\
\hline INTERCEPT & 1.79 & $* * *$ & 0.06 \\
Controls & & & \\
Academic Achievement Index - Math & 0.25 & $* *$ & 0.10 \\
Science/engineering hobbies/activities & 0.09 & $* * *$ & 0.02 \\
Read/watched science/engineering programs & 0.13 & $* * *$ & 0.01 \\
Family interest in science & & \\
High School Physics Sustainability Topics & Included & & \\
Energy supply & 0.12 & $* *$ & 0.04 \\
Sustainable development & 0.15 & $*$ & 0.07 \\
Life cycle analysis & -0.16 & $*$ & 0.12 \\
Bio-mimicry & -0.33 & $* * *$ & 0.09 \\
\hline Adjusted R ${ }^{2}$ & $\mathbf{0 . 2 7}$ \\
\hline
\end{tabular}

Table II. Multiple regression model predicting physics identity proxy with sustainability-related topics in high school physics

\begin{tabular}{llll}
\hline Parameters & Estim. & Sig. & SE \\
\hline INTERCEPT & 0.62 & $* * *$ & 0.11 \\
Controls & & & \\
Gender (0=Male, 1=Female) & -0.38 & $* * *$ & 0.05 \\
Academic Achievement Index - Science & 1.53 & $* * *$ & 0.22 \\
Academic Achievement Index - Math & 0.45 & $*$ & 0.21 \\
Academic Achievement Index - English & -0.82 & $* * *$ & 0.20 \\
Tinkered with things (e.g. motors) & 0.06 & $* *$ & 0.02 \\
Science/engineering hobbies/activities & 0.16 & $* * *$ & 0.02 \\
Read/watched science/engineering programs & 0.06 & $* * *$ & 0.02 \\
Family interest in science & Included & & \\
High School Physics Sustainability Topics & & & \\
Energy supply & 0.16 & $* *$ & 0.05 \\
Food availability & 0.25 & $*$ & 0.13 \\
Bio-mimicry & -0.37 & $* *$ & 0.13 \\
Providing opportunities for future generations & 0.16 & $*$ & 0.07 \\
\hline Adjusted R ${ }^{2}$ & $\mathbf{0 . 2 9}$ & & \\
\hline
\end{tabular}

\section{B. Sustainability}

A significant positive relationship was found for energy supply and sustainable development in the science agency belief model, while energy supply, food availability, and opportunities for future generations were significant and positive for the physics identity model. A significant negative relationship was found for the topic of biomimicry (solutions to human challenges by emulating patterns and strategies in nature) in both the agency belief and identity models.

Results of the multiple regression model utilizing science agency belief and physics identity as the 
outcomes are reported in Table I and Table II, respectively.

\section{DISCUSSION}

What place does a discussion of disease have in an introductory high school physics course? In order to address the implications of our results we emphasize the role of introductory physics in providing a foundation for more complex study e.g. a student motivated to study biological physics or biomedical engineering must see the vital role kinematic particle models can play in problem solving around the advanced study of pathogen movement. This bridge between seemingly dissimilar and disconnected topics may be missed by high school students if not made explicit by a physics teacher. Terrorism and war, water supply, bio-mimicry and other sustainability topics lack that clear-cut link to the traditional canon of physics topics as well. Our results indicate that there are some physics teachers, albeit few, who are exposing students to these topics in physics which lends credence to the fact that perhaps these connections can be made. Unlike these topics energy is a central topic in the high school physics curriculum. However, physics courses are still behind chemistry and biology courses in discussing energy supply - it is discussed in less than one half of high school physics courses according to student reports on the SaGE survey. In addition, most other sustainability topics are also discussed less.

We also see significant value in the findings where activities associated with STEM and family support predicted the outcomes. Thus, beyond the effect of these alternative hypotheses, certain sustainability topic coverage predicts science agency beliefs and physics identity. While this clearly indicates a possible opportunity through these topics, understanding how best to implement lessons around them tis another study in itself. Operating on the premise that sustainability topics carry intrinsic social value, the importance of vitalizing their coverage in any science course can be argued. Further, given that students are experiencing alienation from their physics courses [11] we can posit that an increase in

[1] A. Bartlett, in The essential exponential! For the future of our planet, Edited by R. Fuller, V. Clark, and J. Rogers (University of Nebraska, NE, 2004).

[2] U. Kessels, M. Rau, and B. Hannover, British Journal of Educational Psychology 76, 761-780 (2006).

[3] A. Diekman, E. Brown, A. Johnston, and E. Clark, Psychological Science 21, 1051-1057 (2010).

[4] Z. Hazari, G. Sonnert, P. Sadler, and M. Shanahan, Journal of Research in Science Teaching 47, 978-1003 (2010).

[5] A. Bartlett, Population \& Environment 16, 5-35 (1994).

[6] Sustainability. In United States Environmental Protection Agency. Retrieved from

http://www.epa.gov/sustainability/basicinfo.htm sustainability topic coverage could have a positive impact on physics course enrollment by enhancing science agency beliefs and physics identity.

Finally, we hypothesize that the significance of the negative relationship involving bio-mimicry in both regression models and life-cycle analysis in the first model can be better explained by interactions with other variables. Note that biology-related topics were the only predictors that had a negative relationship. It may be that students who do not associate physics with biology are turned off when exposed to these topics in physics. Thus, there ideas about physics and even biology are challenged. An interaction of the coverage of biomimicry or life-cycle analysis with students' physics epistemologies might reveal why this relationship was observed. We plan to study this further in the future.

While this study is correlational, it is a first step to thinking about re-conceptualizing physics with more integrated topics that address issues of global importance. Future investigations will further our understanding of how sustainability topics can be covered in a physics class and the causal implications of such coverage for students' persistence and performance. Additionally, future investigations of physics teachers' agency beliefs are also appropriate for understanding why such topics are or are not emphasized. We move forward, however, with our own "agency belief" hypothesis. If acquiring knowledge about sustainability through science can provide a path to help students believe they can positively impact the world in which they live, then they are more likely to study science, hopefully physics. When teachers are equipped with this understanding of their students then they can become prime motivators for student success.

\section{ACKNOWLEDGEMENTS}

Thanks to the SaGE participants and team. This material is based on work supported by the National Science Foundation under Grant No. 1036617. Any opinions, findings, conclusions, or recommendations do not necessarily reflect the views of the National Science Foundation.

[7] J. Sullivan, Journal of Research in Science Teaching 45, 10031020 (2008).

[8] S. Basu, A. Barton, N. Clairmont, and D. Locke, Cultural Studies of Science Education 4, 345-371 (2009).

[9] Z. Hazari, G. Sonnert, P. Sadler, and M. Shanahan, Journal of Research in Science Teaching 47, 978-1003 (2010).

[10] R Core Team (2014). R: A language and environment for statistical computing, R Foundation for Statistical Computing, Retrieved from http://www.R-project.org/

[11] U. Kessels, M. Rau, and B. Hannover, British Journal of Educational Psychology 76, 761-780 (2006). 